\title{
ENGINEERED CONTAINMENT AND CONTROL OF AIRBORNE NANOPARTICLES: CURRENT STATUS
}

\author{
STÉPHANE HALLÉ, SYLVIE NADEAU \& JULIEN FATISSON \\ Génie Mécanique, École de Technologie Supérieure, Canada.
}

\begin{abstract}
Industry began years ago to manufacture engineered nanoparticles (NPs) and introduce them into products and processes. Meanwhile, the question of the risks associated with nanotechnologies remains unanswered. International organizations that monitor these risks are recommending not only total containment of NPs but also an integrative approach to achieving this by design. Techniques such as electrostatic precipitation, filtration, wet scrubbing and mechanical separation are effective at containing or extracting airborne NPs and thus minimizing worker exposure. Each of these techniques has its advantages and limitations. This literature review shows that the development of effective NP containment and control technologies would benefit from proper engineering of the manufacturing system as a whole.
\end{abstract}

Keywords: containment; control; engineered nanoparticle; risk management.

\section{INTRODUCTION}

Industry began years ago to manufacture engineered nanoparticles (NPs) and introduce them into products and processes. Based on the latest official world figures, there are about 1814 consumer products currently containing input from nanotechnologies [1]. However, mentioning NP content on the product label is mandatory only in Europe and only for cosmetics and pesticides for the moment. This requirement has been extended to food products in December 2014 [2]. The risks associated with nanotechnologies remain poorly defined and some people find this unsettling. The Trans Atlantic Consumer Dialogue has undertaken to pressure authorities and governments to provide a framework for regulating the consumer health and safety aspects of nanotechnology-based products [3], whereas the French national agency for dietary, environmental and workplace health and safety (ANSES) wishes to reinforce the nanotechnology regulatory framework [4].

Inhalation in the workplace is considered the most probable route of exposure to NP. The hypothesis underlying most containment-based and engineered measures of risk control proposed in the literature is that NPs inevitably become airborne and then behave according to the laws of aerosol physics and conventional fluid mechanics [5, 6]. No model has yet been developed that describes NP aerosol behavior adequately. The NP concentration in the air changes because some of them readily deposit on practically all surfaces. In addition, as Brouwer [7] points out, airborne NPs in the workplace tend to associate with each other and with background particles, thus creating aerosols composed of aggregates and agglomerates, which will sediment more rapidly, thereby reducing exposure via inhalation. Workers nevertheless remain exposed, and containment of airborne NP remains necessary. This article therefore presents a concise overview of the known means of airborne particle containment based on extraction and filtration, as well as their advantages and limitations in the context of the airborne NP problem. 


\section{INSIGHTS OF CURRENT REGULATORY FRAMEWORK}

Since the individuals exposed first and foremost to the products of nanotechnology are workers $[8,9]$ and the numbers of such workers are increasing as markets continue to expand, several organizations have raised concerns regarding the current void in nanotechnology product regulation, characterization and health impact and have made known their recommendations:

- Mandatory registration, evaluation and authorization procedures [10-17]. France was the first country to require NP-producing industries to declare annual production, beginning in 2013. According to the Ministry of Sustainable Development, the figure for 2013 reached just over 420,000 metric tons of NP materials produced in or imported into France [18].

- Identification and characterization of risks, physical and chemical properties, and exposure at each stage of the product life cycle [11, 15, 19, 20]. Given the inconsistencies in the few existing regulations in the world of nanotechnologies, Europe and the USA have signed an agreement to guide a concerted effort to implement precise and effective measures for managing the risks associated with NP production and use [21].

The International Standards Organization (ISO) has produced so far over 60 documents on the subject of standardization with regard to nanotechnologies. These cover proper definitions and nomenclature as well as recommendations regarding toxicity tests but mostly various aspects of characterization tests. ISO has also published recommendations regarding voluntary labeling of nanotechnology-based consumer products [22] since no law currently obliges manufacturers to do so, except for the European law covering only cosmetics for the moment. Meanwhile, the European Parliament has rejected a bill that would have limited the mandatory labeling obligation for consumer products containing nanotechnology input [23].

With regard to the control of exposure to NP through inhalation, ISO standard 12901 is worthy of particular attention. The first part of this standard [24] deals with workplace risk management and indicates NP control measures, while the recently published second part [25] recommends the use of a control banding approach to manage different levels of risk. ISO standard 12901-1:2012 completes, adds detail to and updates ISO standard 12885 [26] which, in addition to defining NP materials and proposing characterization standards and exposure tests, proposes various control measures starting at risk evaluation, followed by engineering (e.g. suitable ventilation), and on up to administrative measures. It also suggests implementing exposure prevention measures that are specific for each NP type and each task when faced with persistent uncertainties surrounding product characterization and the effectiveness of control measures. For the work environment specifically, ISO standard 12901-1 suggests a complete framework for the management of occupational risk: (1) identifying and evaluating risks; (2) deciding what precautions are necessary; (3) adequate avoidance or control of exposure; (4) ensuring that control measures are applied and maintained; (5) monitoring exposure; (6) carrying out suitable monitoring of worker health; (7) preparing plans and procedures to follow when incidents, accidents or emergencies occur and (8) ensuring that personnel are properly informed, trained and supervised.

In the particular case of control measures, a hierarchy of measures is proposed through various documents published by workplace health and safety monitoring organizations $[5,6$, $11,13,15,20,24,26-28,30,31]$. This hierarchy is conceptualized in Figure 1. These schematics categorize the different means of action available to prevent the exposition to NPs as a function of control from intrinsic to individual protection. As shown in the literature, eval- 


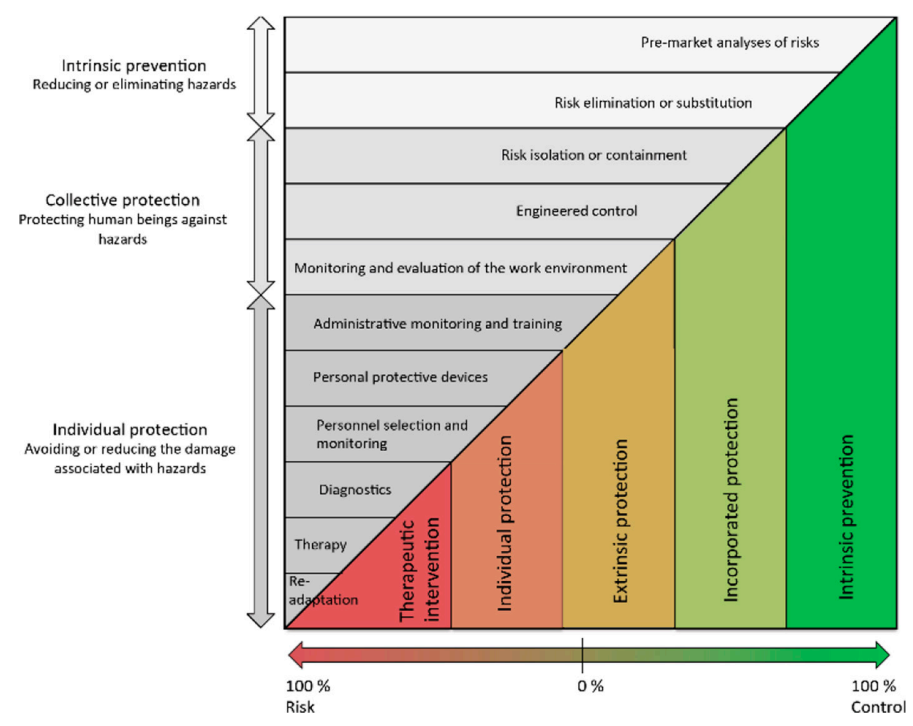

Figure 1: Hierarchy of control measures applicable to NP production (conceptualization based on published literature).

uation of risks and risk elimination are the most effective whilst readaptation and therapy are the most riskful measures to contain workers' exposure from harmful chemicals.

\section{SOURCES OF NP AND ENGINEERING CONTROL}

It is recommended in the literature more specifically that NP be confined and contained through manufacturing system design $[26,28,30,32]$ as well as during system operation and maintenance. Special attention is needed where maintenance, handling and packaging personnel are involved, since ergonomically poor postures increase the risk of losing control of loads [33]. Similar attention is needed in any situation where accidental spilling or air dispersal of NP could occur [5, 6, 33]. Airborne or air-formed NPs are likely to reach significant concentrations during certain phases of production $[34,35]$, handling of NP powders (packaging of product and cleaning of reactors) $[34,36]$ or processing of finished products [34]. These observations underscore the importance of using engineering principles to determine the effectiveness of the means applied to controlling exposure.

The applicability and effectiveness of means of control of exposure to NP aerosols depend on mechanisms that affect the aerodynamic behavior of NP in air. Current understanding of the physicochemical properties of NP aerosols on or in exposed workers remains limited. Figure 2 provides a schematic representation of the principal mechanisms affecting the concentration, morphology, size distribution and aerodynamic behavior of NPs in aerosols at the source and around workers. These mechanisms affect the NP aerodynamic behavior as well as their deposition in the human respiratory tract, and hence the degree of actual exposure. In addition, the toxicity of the NP themselves depends on some of these mechanisms. Since there is still no consensus regarding the metric to use for the evaluation of exposure to NP, monitoring and measurement in the workplace involve evaluation of the specific surface, determination of particles mass and number concentrations, shape factor and chemical composition also. 


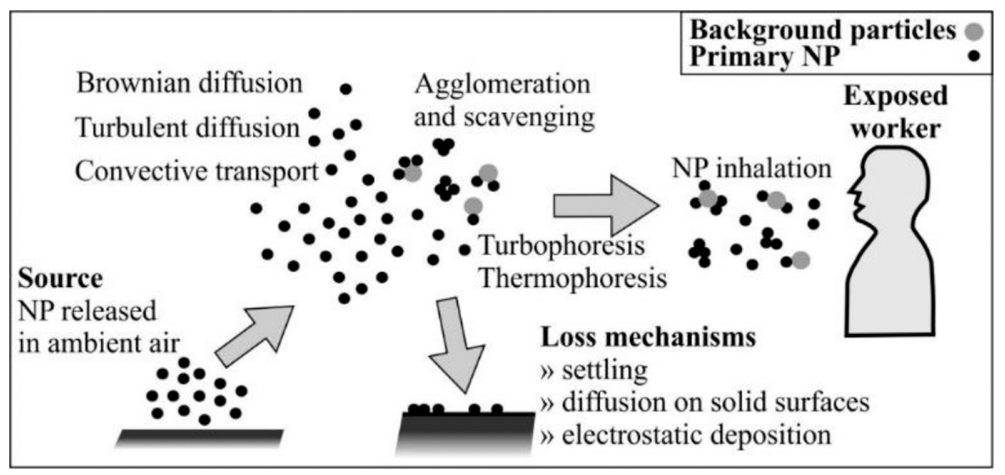

Figure 2: Schematization of various mechanisms affecting airborne NP concentration.

Businesses that produce NP are not the only workplaces where exposures may be considered significant. Research laboratories are another source of airborne NP. In a review, Kuhlbusch et al. [34] listed the operations during which NP may become airborne and which therefore require containment, whether in research laboratories or in NP-producing industrial businesses. These authors suggest taking into consideration both these environments to evaluate and predict exposure to NP with better accuracy. In both cases, containment of NP must be complete, in a room under a negative pressure differential relative to adjoining rooms [37, 38]. Local or central systems of ventilation or extraction may be adapted to maximizing NP trapping as long as they are fitted with appropriate filters. The ISO currently recommends using high-efficiency particulate or high-efficiency particulate (HEPA) filters [24, 26, 30, 31, 39]. Systems for filtering effluent water potentially containing NP must be fitted with filters or brushes for extracting water-soluble organic matter [39]. According to the NIOSH report published in 2012, almost all of the producers of carbon-based NP materials queried a few years earlier were applying ventilation-based means of control as prescribed or were applying even more effective measures [31]. More than half of these companies were also using local extraction systems.

A review of actual field practice [10, 15, 33, 36, 39-42] reveals that NP production and handling practices vary considerably. This variation appears to be due to factors such as the cost associated with preventive measures, the cost of the NP supply, the lack of information and consistent guidelines. Several organizations including the Institut de Recherche Robert-Sauvé en Santé et en Sécurité du Travail (IRSST) have produced guides describing good industrial practices [29], and the low priority given to addressing workplace health and safety issues, particularly in contexts where regulation is absent and standardization is deficient [39]. Although the short-term cost of installing an engineered exposure control system may appear daunting in comparison with the cost of relying on administrative controls and protective equipment, long-term savings may be realized if the system is properly designed [38]. Among the dubious practices in the realm of preventive measures, we note in particular the following:

- Relying on glass barriers to limit exposure of workers to NP being handled in flow hoods that are shut off to avoid loss of material [39];

- Incineration of waste that may contain NP [10] that could become airborne and dispersed in combustion gases and soot. 


\section{LITERATURE SEARCH METHOD}

The search was carried out according to a previously described and commonly adopted systematic protocol $[43,44]$. Using search tools such as PubMed and Scopus, we conducted a systematic review of literature published up to the present by entering various combinations of the following keywords: nanoparticle, nanomaterial, nano, containment, control measures, removal and extraction. Review articles and reports by commissions or by organizations such as NIOSH were examined first. The references cited in these documents were used to identify additional studies. To narrow down the results, a descending method based on recent bibliographies was used with keywords such as 'wet collector', 'wet scrubber', 'filtration', 'electroprecipation', 'separation' or 'cyclone' in various combinations. We were thus able to gather information on the principles, advantages and limitations of the various methods of airborne NP containment. Some articles were excluded mostly on the basis of the subject matter in comparison with the research question, thus reducing interpretation biases and deficient research protocols.

\section{CURRENT MEANS OF NANOPARTICLES CONTAINMENT}

The use of central ventilation systems to control exposure to NP is generally not recommended [31]. If the quantity of NP in the air is too large, the airflow rate required to ensure adequate dilution may be greater than the system is capable of delivering. In addition, ventilation will not provide a sufficient reduction in airborne NP concentration if the distance between the emission source and the worker is short or if particle toxicity is high. Finally, to make central ventilation effective, dispersion of the airborne NP must be relatively uniform and not limited to a few separate locations $[31,45]$. According to NIOSH studies, the best way to protect workers by means of ventilation is to use local extraction systems, of which the effectiveness has been demonstrated for various airborne NP-generating operations [36, 46].

There are four principal means known to be effective for particle extraction and filtration from air [47-49] (Fig. 1):

- Contact with water. This involves contacting the air with water vapor to surround the particles with water molecules. Deflectors then guide the water-surrounded particles to a holding tank. The contaminated water is pumped out and filtered. These devices are called wet scrubbers or wet collectors.

- Contact with a filtering medium. HEPA filters and numerous textile filters are suitable. According to the single-fiber filtration model, electrostatic attraction, diffusion or polarization forces hold NP aggregates that are below $200 \mathrm{~nm}$ in size while particles $300 \mathrm{~nm}$ or larger are retained by the filter due to impaction, interception and gravitational sedimentation mechanisms [50]. These filters are effective by virtue of one or more filtration mechanisms depending on airborne NP size and static charge (to name a few of the essential characteristics), whether via diffusion, interception, inertial impact, gravitational sedimentation or even electrostatic attraction [51].

- Electrostatic precipitation. In this case, the air is forced through a passage in which an ionizing discharge imparts a negative electrical charge to the particles [48]. The charged particles are then attracted to plates charged positively by high voltage. The plates are washed regularly using jets of hot water and friction.

- Mechanical separation. Cyclones, gravity chambers or impact separators can be used to concentrate and guide particles toward a sedimentation chamber. This is achieved by creating a vortex in the fluid (air) inside a conical chamber, by circulation of the fluid inside a 
chamber that has a large surface area and decreases its speed, or by dividing the fluid into several high-speed streams that pass by barriers while the particles remain on a linear path and strike these barriers, which are coated with NP-retaining adhesive materials. These separators may also be combined with electrostatic precipitators using electrically charged plates instead of adhesive barriers [52].

In addition to these four widely practiced means, there are two other methods that are used much less but do allow removal of NP from volumes or surfaces. Dry scrubbing systems consist of a forced flow reactor and a subsequent collection device, e.g. a bag filter in which both particulate matter and gaseous components are collected simultaneously [48]. Removal of NP using laser-induced plasma has also been described [53-54], but this appears applicable to the cleaning of small surfaces, making it an unlikely candidate for industrial-scale use in a NP manufacturing company.

\section{DISCUSSION}

\subsection{Advantages and limitation of known means of NPs extraction and filtration}

Although each method of particle extraction or air filtration has its limitations even outside of its specific application to engineered NPs, the advantages of each need to be appreciated as well $[47,49,55]$. Table 1 summarizes these various characteristics and shows that much remains to be determined about the actual performance of these different means of particle containment.

NP aerosols in the workplace are generally transported in the ambient air by convection and diffusion [56]. Since inertial forces acting on particles are proportional to particle mass, these forces are very weak for NP, which therefore follow fluid streamlines very closely. The ineffectiveness of inertial wet scrubbers [57-58] provided motivation to develop systems incorporating electrostatic precipitation, thus combining the advantages of both methods and eliminating some of the problems with conventional systems. However, these systems are still in development [59] and cannot at the present time replace either of the other two, since they require coupling with existing systems such as filtration. They are not economical in terms of energy or materials and offer little to solve the dilemma of the compromise between the cost of manufacturing and the quality of the workplace environment [48].

In the case of filters, effectiveness is defined in terms of the most penetrating particle size (MPPS). This parameter depends on several parameters: airflow speed, the filtration mechanism, filter type, particle type, particle net charge and the charge density of the fibers [51, 60]. This multifactorial dependence makes the certification of filters relatively complex. A NIOSH document published recently [38] shows a graph of the efficiency of various types of filter. For particles smaller than $100 \mathrm{~nm}$, diffusion filters (e.g. HEPA) are more efficient than interception or impact filters, which are more efficient for particles larger than $500 \mathrm{~nm}$. The MPPS is defined as the average aerodynamic mass diameter. It should also be noted that the size measurement method also influences the determination of filter performance. Mostofi et al. [61] have shown that the MPPS of a given NP for a given filter differs for electrical-mobility-based and inertia-based measurements.

\subsection{The complexity of the nanoparticles case}

There are various types of engineered NP: nanostructured bulk materials, surface-nanostructured materials, materials containing nanostructured particles bound to surfaces (19\%), 


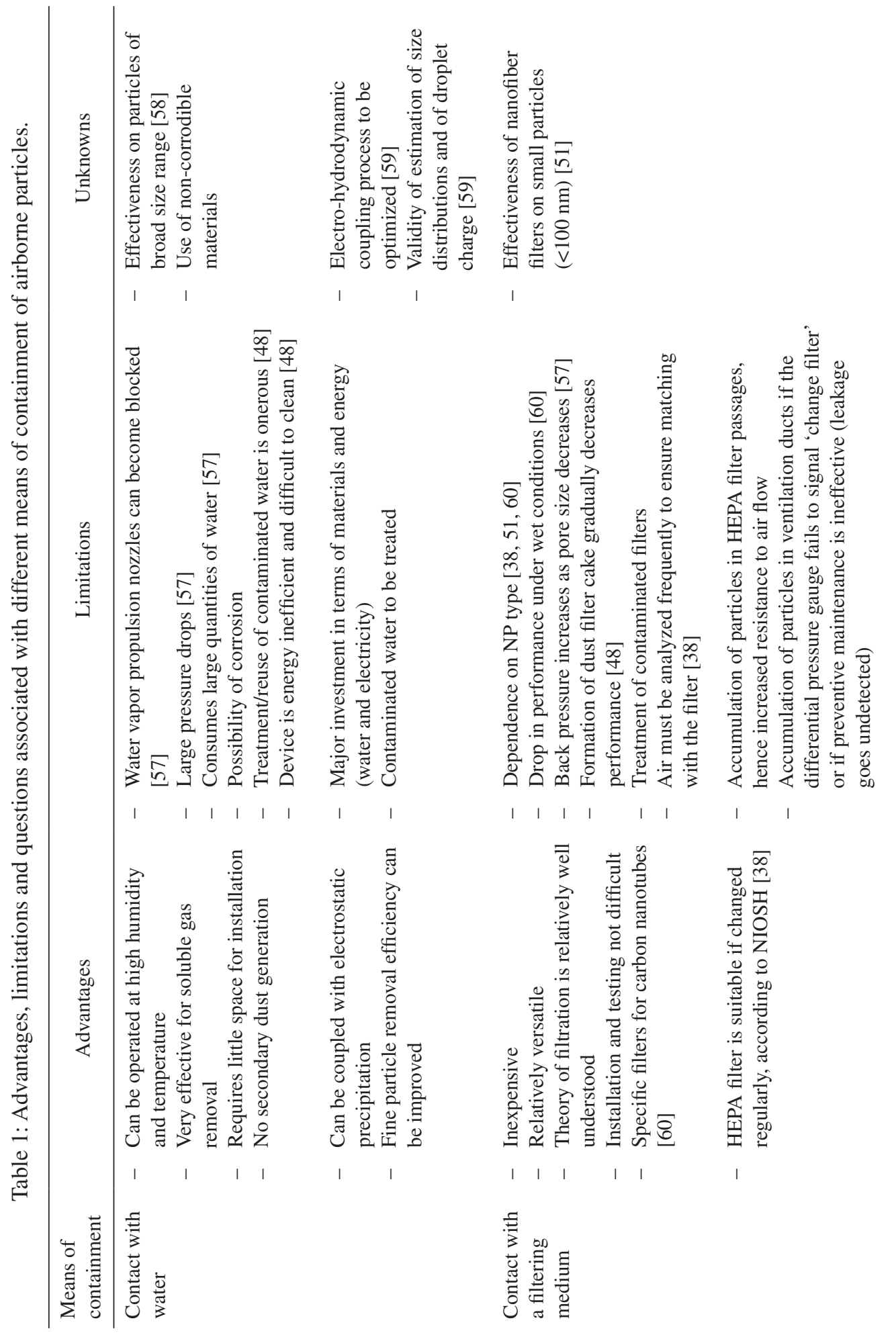




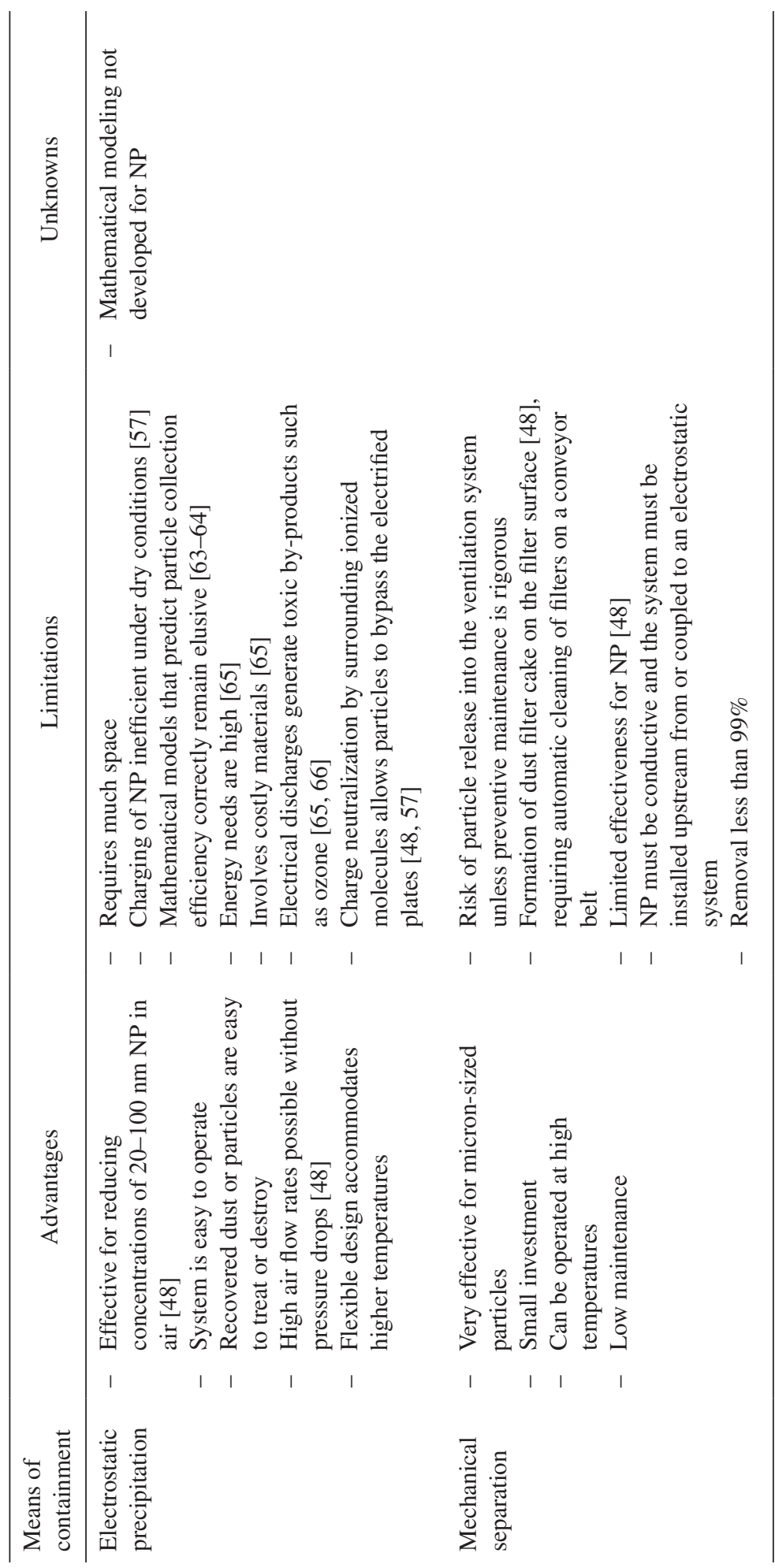


suspended in liquid (37\%), suspended in a solid (13\%) and airborne (1\%) [67]. There are also various NP manufacturing processes: ball milling, etching, sonication, laser ablation, sol-gel, chemical vapor deposition, plasma or flame spraying, supercritical fluid, spinning and self-assembly [67]. The multiplicity of materials and processes makes the generalization of control measures almost impossible or at least requires the development of complex mathematical models to assess the risks.

Mazzuckelli et al. [40] published the findings of an inquiry in a nanostructured composite materials synthesis laboratory (carbon nanofibers). Their research protocol included observation, semi-directed interviews, toxicological sampling, transmission electron microscopy analysis, visual inspection of the ventilation system and extraction tests with tracer gases. They were unable to distinguish between endogenous (i.e. synthesis or manufacturing processes) and exogenous sources of NP or conclude that the zones of concentration measured corresponded to the actual concentrations in the workers' inhalation zone.

Filtration theory suggests that NP retention in filters should increase as particle size decreases. However, this appears not always to be the case, and below a very small size $(1 \mathrm{~nm})$, NP tend to behave essentially as molecules and appear to be affected by a thermal rebound effect that causes them to detach from the filter fibers [51]. Givehchi and Tan [68] have devoted a study to the matching of currently used mathematical models with experimental results and noted that the validity of the single fiber model as a theory of filtration is weak and that the model does not take into consideration all of the phenomena that come into play.

Compared with their micron-sized homologues, engineered NPs tend to agglomerate quickly. The behavior of these agglomerates in filtration media has been studied very little to date [69]. They are partly responsible for the formation of 'dust cakes' on the surface of filters and the resulting reduced performance.

In view of the lack of consensus regarding suitable metrics for evaluating airborne NP, it is difficult to define adapted or adaptable standards of NP characterization. Such standards are nevertheless necessary since the particle type tested influences filter performance [70]. It should be noted that current testing for filters used in breathing apparatus is carried out with sodium chloride NPs in accordance with NIOSH recommendations [71] even though using such particles as a standard can be questionable since they are usually not used in industrial processes. Efforts are underway to find new methods for evaluating filter media performance with NP specifically while taking into consideration the theory of the various filtration mechanisms that may be involved [72].

There is a need for standardization of tests and testing methods. In spite of the existence of several standards for nanotechnologies and their use, there is still no document devoted to standardization of methods of evaluation of sprays containing NP [73]. This might explain why one of the major challenges in terms of measuring exposure using different techniques remains taking into consideration background NPs, which may be difficult to distinguish from NPs generated by reactors [34, 74].

Reaching a conclusion thus becomes complicated since filters are not tested with the same NPs or under the same filtration conditions (flow rate, moisture level, etc.). This difficulty in reaching a consensus to facilitate comparisons is maintaining a structural uncertainty throughout the entire techno-economic system within which nanotechnologies are evolving [75].

\subsection{In the current context, what engineered control measures should be considered?}

The effectiveness of engineered control measures proposed in the literature has seldom been validated using an experimental approach. The standards of proof as they are generally 
applied in science have not been yet imposed in all cases. It therefore becomes necessary to subject our hypotheses to the process of rejection or acceptance, in other words to measure their consistency with experienced reality. Scientific method is used to identify hypotheses that may be rejected on the basis of inconsistency with facts or existing knowledge. The most plausible hypotheses can be chosen even in uncertain situations [76].

Demou et al. [77] confirmed that the production technique has an impact on the quantity and size of the NP obtained. Proper engineering at the equipment design stage therefore could potentially enhance NP containment efficiency at the point of production.

It should be noted that the design of the NP extraction system has a considerable effect on system efficiency. Constant-rate laminar flow systems appear to provide the most variable performance while air curtains of more recent design appear to be the most effective for reducing worker exposure [78]. A relatively recent NIOSH document provides the details of a study of each type of flow hood or cabinet [30]. In situations involving airborne NP, the NIOSH recommendation is for total containment in a glove box or other sealed chamber connected to an extraction system fitted with HEPA filters. The ventilation system must be adapted specifically to the production system [79], which requires an integrated approach starting at the design stage of a nanomaterial manufacturing business. Based on current knowledge in fluid mechanics, unidirectional airflow should be preferred to avoid NP dispersal throughout the work environment. According to the NIOSH guide, the air of rooms in which NPs are produced or handled should be renewed completely 4-12 times per hour and the air pressure should of course be negative with respect to all adjoining rooms, passages or ducts [30].

In the absence of sufficient knowledge on the toxicity, flammability, mass and possible phase changes of NP at different temperatures, the use of central ventilation systems to dilute the NP concentration should be avoided, and contaminated air should not be vented to the environment [30, 38].

NP control measures not associated with ventilation include the use of additives and barriers, which can be combined with ventilation to increase the effectiveness of protection thus provided [38]. HEPA filters can drastically reduce exposure to NP. However, some questions remain on their efficiency for particles in the single-digit nanometers range. Small NPs may rebound from the filter surface if their kinetic energy is high enough to overcome the adhesion energy. Until now, published studies have not shown whether this effect is important or not [68]. The inevitable steady decline in HEPA filter effectiveness and performance must also be managed properly with regular replacement [80] and disposal procedures for HEPA filters must be in place.

Workers' occupational exposure assessment and routine monitoring for airborne NP should be performed so as to be able to detect leak or malfunction of control systems. To reach that goal, real-time instruments should be used since they can trigger alarms in the case of accidental spill. Although several portable instruments have developed for this task [81, 82], they have their shortcomings. Moreover, the time resolved instruments cannot distinguish between the engineered airborne NPs from background NPs. A small amount of released NPs may not be detected if the number concentration is similar to the background noise concentration.

\section{CONCLUSIONS}

To ensure that measures implemented to prevent worker exposure to engineered NPs are matched on the long term with the properties (size, charge, shape, etc.) of airborne and atmospherically formed NP, continuous evaluation is strongly recommended. There is no consensus 
yet on the proper metric to use to evaluate exposure. A popular choice appears to be the use of a plurality of metrics: mass concentration, volumetric concentration and size distribution. The question remains open because of the inherent complexity of the systems, in which risk factors are interdependent and properties are influenced by the nature of the particles involved. The most recommendable approach to achieving sustainability of nanotechnology development appears to be a comprehensive and integrative one in which particles and exposures thereto are fully characterized, pressure drops across filters are monitored and protective measures are properly engineered.

This literature review shows that the development of effective NP containment and control technologies requires proper engineering of the manufacturing system as a whole. However, this raises questions regarding the recycling and disposal of fouled filters and the treatment of contaminated water and spent process oils. Machine designers and occupational health and safety practitioners will have to keep this type of question in mind on the long term. By integrating all aspects of risk over the entire industrial process lifecycle, better protection of both workers and the environment may be expected.

\section{REFERENCES}

[1] Project on Emerging nanotechnologies, Consumer product inventory, 2015, available at http://www.nanotechproject.org/cpi/products/ (consulted 23 February 2015).

[2] VeilleNanos, Plus de produits contenant des nanomatériaux sur le marché: quelques chiffres ... et beaucoup de questions, 2013, available at http://veillenanos.fr/wakka.php ?wiki=PenNanoInventoryMajOct2013\#Cpi (consulted 2 June 2014.).

[3] TransAtlantic Consumer Dialogue, Nanotechnologies Overview, 2014, available at http://tacd.org/nanotechnologies/ (consulted 2 June 2014).

[4] ANSES, Highlighting the toxicity of certain nanomaterials, ANSES is calling for a stronger regulatory framework, published 15 May 2014, available at http://www.anses. $\mathrm{fr} / \mathrm{en} / \mathrm{content} / \mathrm{highlighting-toxicity-certain-nanomaterials-anses-calling-stronger-regu-}$ latory-framework (consulted 5 June 2014).

[5] Schulte, P.A., Geraci, C.L., Zumwalde, R., Hoover M. \& Kuempel, E., Occupational risk management of engineered nanoparticles. J. Occup. Environ. Hyg., 5, pp. 239-249, 2008. doi: http://dx.doi.org/10.1080/15459620801907840

[6] Schulte, P.A. , Trout, D., Zumwalde, R.D., Kuempel, E., Geraci, C.L., Castranova, V., Mundt, D.J., Mundt, K.A. \& Halperin, W.E., Options for occupational health surveillance of workers potentially exposed to engineered nanoparticles: state of science. J. Occup. Environ. Med., 50, pp. 517-526, 2008. doi: http://dx.doi.org/10.1097/JOM.0b013e31816515f7

[7] Brouwer, D., Exposure to manufacture nanoparticles in different workplaces. Toxicology, 269, pp. 120-127, 2010. doi: http://dx.doi.org/10.1016/j.tox.2009.11.017

[8] Maynard, A.D. \& Pui, D.Y.H., Nanotechnology and occupational health: new technologies-new challenges. J. Nanopart. Res., 9, pp. 1-3, 2007. doi: http://dx.doi.org/10.1007/ s11051-006-9164-8

[9] Schulte, P.A., Geraci, C.L., Murashov, V., Kuempel, E.D., Zumwalde, R.D., Castranova, V., Hoover, M.D., Hodson, L. \& Martinez, K.F., Occupational safety and health criteria for responsible development of nanotechnology. J. Nanopart. Res., 16, pp. 1-17, 2014. doi: http://dx.doi.org/10.1007/s11051-013-2153-9

[10] Gamo, M. \& Kishimoto, A., Current practices of risk management for nanomaterials by companies in Japan. Report of findings: Research Project on Facilitation of Public Acceptance of Nanotechnology, 2006, 13 pp. 
[11] Rickerby, D.G., Nanotechnological medical devices and nanopharmaceuticals: the European Regulatory Framework and research needs. J. Nanosci. Nanotechnol., 7, pp. 4618-4625, 2007.

[12] Balbus, J.M., Maynard, A.D., Colvin, V.L., Castranova, V., Daston, G.P., Denison, R.A., Dreher, K.L., Goering, P.L., Goldberg, A.M., Kulinowski, K.M., Monteiro-Riviere, N.A., Oberdörster, G., Omenn, G.S., Pinkerton, K.E., Ramos, K.S., Rest, K.M., Sass, J.B., Silbergeld, E.K. \& Wong, B.A., Meeting report: hazard assessment for nanoparticles - report from an Interdisciplinary Workshop. Environ. Health Perspect., 115, pp. 1654-1659, 2007. doi: http://dx.doi.org/10.1289/ehp.10327

[13] European Commission (a), 1st Annual Nano Safety for Success Dialogue, Community Health and Consumer Protection, Brussels, 25-26 October 2007, 26 pp.

[14] Government of Canada, Proposed Regulatory Framework for Nanomaterials under the Canadian Environmental Protection Act, 1999, 2007.http://www.ec.gc.ca/substances/ nsb/eng/nanoproposition_e.shtml

[15] Wetzel, M.S., Environmental, health and safety issues and approaches for the processing of polymer nanocomposites, 66th Annual Technical Conference of the Society of Plastics Engineers, Milwaukee, Wisconsin, USA, 4-8 May, 2008, pp. 247-251.

[16] Haut Conseil de la Santé Publique, Avis relatif à la sécurité des travailleurs lors de l'exposition aux nanotubes de carbone, Ministère de la Santé, de la Jeunesse, des Sports et de la vie Associative, France, 2009, 10 pp.

[17] US Environmental Protection Agency, Regulating pesticides that use nanotechnology, 2011, Consulted June 2, 2014. available at http://www.epa.gov/pesticides/ regulating/ nanotechnology.html\#application.

[18] Ministère du Développement Durable, Rapport d'étude sur les éléments issus des déclarations des substances à l'état nanoparticulaire, 2013, available at http://www.developpement-durable.gouv.fr/IMG/pdf/Rapport_public_format_final_20131125.pdf

[19] Friedrichs S. \& Schulte, J., Environmental, health and safety aspects of nanotechnology - implications for the R\&D in (Small) Companies, Sci Technol. Adv. Mater., 8, pp. 12-18, 2007. doi: http://dx.doi.org/10.1016/j.stam.2006.11.020

[20] Warheit, D., Sayes, C.M., Reed, K.L. \& Swain, K.A., Health effects related to nanoparticle exposures: environmental, health and safety considerations for assessing hazards and risks. Pharmacol. Ther., 120, pp. 35-42, 2008. doi: http://dx.doi.org/10.1016/j. pharmthera.2008.07.001

[21] Ministère des Affaires Étrangères, Gestion des risques liés aux nanotechnologies. Une coopération Europe-Etats-Unis, 2013, available at http://www.bulletins-electroniques. com/actualites/74320.htm (consulted 2 June 2014).

[22] International Organization for Standardization, ISO/TR 13830:2013. Nanotechnologies - Guidance on Voluntary Labelling for Consumer Products Containing Manufactured Nano-objects, Milwaukee, Wisconsin, USA, 4-8 May, 2013, 6 pp.

[23] Nano and Other Emerging Technologies Blog, EU Amends Food Labeling Regulation Concerning Definition of Engineered Nanomaterials, 2013, available at http://nanotech.lawbc.com/2013/12/articles/international/eu-amends-food-labeling-regulationconcerning-definition-of-engineered-nanomaterials/ (consulted 2 June 2014).

[24] International Organization for Standardization, ISO/TR 12901-1:2012. Nanotechnologies - Occupational Risk Management Applied to Engineered Nanomaterials - Part 1: Principles and Approaches, Milwaukee, Wisconsin, USA, 4-8 May, 2012, 37 pp. 
[25] International Organization for Standardization, ISO/TS 12901-2:2014. Nanotechnologies - Occupational Risk Management Applied to Engineered Nanomaterials - Part 2: Use of the Control Banding Approach, 2014, 31 pp.

[26] International Organization for Standardization, ISO/TR 12885:2008. Nanotechnologies - Health and Safety Practices in Occupational Setting Relevant to Nanotechnologies, Milwaukee, Wisconsin, USA, 4-8 May, 2008, 79 pp.

[27] Wiesner, M.R., Lowry, G.V., Alvarez, P., Dionysiou, D. \& Biswas, P., Assessing the risks of manufactured nanomaterials. Environ. Sci. Technol., 40, pp. 4336-4345, 2006. doi: http://dx.doi.org/10.1021/es062726m

[28] Guo, L., Liu, X., Sanchez, V., Vaslet, C., Kane, A.B. \& Hurt, R.H., A window of opportunity: designing carbon nanomaterials for environmental safety and health. Mater. Sci. Forum, 544-545, pp. 511-516, 2007. doi: http://dx.doi.org/10.4028/www.scientific.net/ MSF.544-545.511

[29] Ostiguy, C., Roberge, B., Ménard, L. \& Endo, C.-A., Best Practices Guide to Synthetic Nanoparticles Risk Management, IRSST, R-599, Institut de Recherche Robert Sauvé en Santé et Sécurité au Travail: Montréal, Canada, 2009, 67 pp.

[30] NIOSH (a), General safe practices for working with engineered nanomaterials in research laboratories, Department of Health and Human Services, Centers for Disease Control and Prevention, National Institute for Occupational Safety and Health, DHHS (NIOSH), Publication No. 2012-147, 2012.

[31] NIOSH (b), Filling the knowledge gaps for safe nanotechnology in the workplace. Department of Health and Human Services, Centers for Disease Control and Prevention, National Institute for Occupational Safety and Health, DHHS (NIOSH) Publication No. 2013-101, 2012.

[32] Ostiguy, C., Roberge, B., Woods, C. \& Soucy, B., Les nanoparticules de synthèse Connaissances actuelles sur les risques et les mesures de prévention en SST, 2nd édn. Rapport IRSST R-646, 2010, 159 pp.

[33] Shatkin, J.A. \& Barry, B.E., Approaching risk assessment of nanoscale materials. NSTINanotech, 1, pp. 553-556, 2006.

[34] Kuhlbusch, T.A.J., Asbach, C., Fissan, H., Gohler D. \& Stintz, M., Nanoparticle exposure at nanotechnology workplaces: a review. Part. Fibre Technol., 8, pp. 1-18, 2011. doi: http://dx.doi.org/10.1186/1743-8977-8-22

[35] Hedmer, M., Isaxon, C., Nilsson, P.T., Ludvigsson, L., Messing, M.E., Genberg, J., Skaug, V., Bohgard, M., Tinnerberg, H. \& Pagels, J.H., Exposure and emission measurements during production, purification, and functionalization of arc-discharge-produced multi-walled carbon nanotubes. Ann. Occup. Hyg., 58, pp. 355-379, 2014. doi: http://dx.doi.org/10.1093/annhyg/met072

[36] Old, L. \& Methner, M.M., Engineering case reports: effectiveness of local exhaust ventilation (LEV) in controlling engineered nanoparticle emissions during reactor cleanout operations. J. Occup. Environ. Hyg., 5, pp. D63-D69, 2008. doi: http://dx.doi. org/10.1080/15459620802059393

[37] Hallock, M.F., Greenley, P., DiBerardinis, L. \& Kallin, D., Potential risks of nanomaterials and how to safely handle materials of uncertain toxicity. J. Chem. Health Safety, January-February, pp. 16-23, 2008.

[38] NIOSH, Current Strategies for Engineering Controls in Nanomaterial Production and Downstream Handling Processes, U.S. Department of Health and Human Services, Centers for Disease Control and Prevention, National Institute for Occupational Safety and Health, DHHS (NIOSH), Cincinnati, OH, Publication No. 2014-102, 2013. 
[39] Conti, J.A., Killpack, K., Gerritzen, G., Huang, L., Mircheva, M., Magali, D., Harthorn, B.H., Appelbaum, R.P. \& Holden, P.A., Health and safety practices in the nanomaterials workplace: results from an international survey. Environ. Sci. Technol., 42, pp. 31553162, 2008. doi: http://dx.doi.org/10.1021/es702158q

[40] Mazzuckelli, L., Methner, M.M., Birch, M.E., Evans, D.E., Ku, B-K., Crouch, K. \& Hoover, M.D., Case study: identification and characterization of potential sources of worker exposure to carbon nanofibers during polymer composite laboratory operations. J. Occup. Environ. Hyg., 4, pp. D125-D130, 2007. doi: http://dx.doi. org/10.1080/15459620701683871

[41] Schmid, K. \& Riediker, M., Use of nanoparticles in Swiss industry: a targeted survey. Environ. Sci. Technol., 42, pp. 2253-2260, 2008. doi: http://dx.doi.org/10.1021/es071818o

[42] Methner, M.M., Hodson, L., Dames, A. \& Geraci, C., Nanoparticle emission assessment technique (NEAT) for the identification and measurement of potential inhalation exposure to engineered nanomaterials - Part B: results from 12 field studies. J. Occup. Environ. Hyg., 7, pp. 163-176, 2010. doi: http://dx.doi.org/10.1080/15459620903508066

[43] Boote, D.N. \& Beile, P., Scholars before researchers: on the centrality of the dissertation literature review in research preparation. Educational Researcher, 14, pp. 3-15, 2005.

[44] Randolph, J.J., A guide to writing the dissertation literature review. Practical assessment. Research and Evaluation, 14, 13 pp., 2009.

[45] ACGIH (American Conference of Governmental Industrial Hygienists), Industrial ventilation: a manual of recommended practice for operation and maintenance. American Conference of Governmental Industrial Hygienists, Cincinnati, OH, February 1, 2010.

[46] Mohlmann, C., Welter, J., Klenke, M. \& Sander, J., Workplace exposure at nanomaterial processes. J. Phys. Conf. Series, 170, 5 pp., 2009.

[47] Jennings, B.H., Environmental Engineering. Analysis and Practice. International Textbook Company: Scranton, Pennsylvania, USA, 1969, 765 pp.

[48] Peukert, W. \& Wadenpohl, C., Industrial separation of fine particles with difficult dust properties. Powder Technol., 118, pp. 136-148, 2001. doi: http://dx.doi.org/10.1016/ S0032-5910(01)00304-7

[49] Asfahl, C.R., Industrial Safety and Health Management, Pearson Prentice Hall: USA, 512 pp., 2014.

[50] Schaffer, R.E. \& Rensagamy, S., Respiratory protection against airborne nanoparticles: a review. J. Nanopart. Res., 11, pp. 1661-1672, 2009. doi: http://dx.doi.org/10.1007/ s11051-009-9649-3

[51] Wang, C-S. \& Otani, Y., Removal of nanoparticles from gas streams by fibrous filters: a review. Ind. Eng. Chem. Res., 52, pp. 5-17, 2013.

[52] Lin, G-Y., Cuc, L-T., Lu, W., Tsai, C-J., Chein, H-M. \& Chang, F-T., High-efficiency wet electrocyclones for removing fine and nanosized particles. Sep. Purif. Technol., 114, pp. 99-107, 2013. doi: http://dx.doi.org/10.1016/j.seppur.2013.04.039

[53] Varghese, I., Murthy Peri, M.D., Dunbar, T., Maynard, B., Thomas, D.A. \& Cetinkaya, C., Removal of nanoparticles with laser-induced plasma. J. Adhesion Sci. Technol., 22, pp. 651-674, 2008. doi: http://dx.doi.org/10.1163/156856108X305561

[54] Murthy Peri, M.D., Devarapalli, V. \& Cetinkaya, C., Selective removal of 10-40 nm particles from silicon wafers using laser-induced plasma shockwaves. J. Adhesion Sci. Technol., 21, pp. 331-337, 2007. doi: http://dx.doi.org/10.1163/156856107780684594

[55] Nazaroff, W.W. \& Alvarez-Cohen, L., Environmental Engineering Science, 1st edn., Wiley, Hoboken, New Jersey, USA, 2000, 704 pp. 
[56] Morency F. \& Hallé, S., A simplified approach for modelling airborne nanoparticles transport and diffusion, J Comput. Methods Experiment Measurements, 1, pp. 55-71, 2013. doi: http://dx.doi.org/10.2495/CMEM-V1-N1-55-71

[57] Jaworek, A., Balachandran, W., Krupa, A., Kulon, J. \& Lacrowski, M., Wet electroscrubbers for state of the art gas cleaning. Environ. Sci. Technol., 40, pp. 6197-6207, 2006. doi: http://dx.doi.org/10.1021/es0605927

[58] Jaworek, A., Krupa, A., Sobczyk, A.T., Marchewicz, A., Szudyga, M., Antes, T., Balachandran, W., Di Natale, F. \& Carotenuto, C., Submicron particles removal by charged sprays. Fundamentals. J. Electrostatics, 71, pp. 345-350, 2013. doi: http:// dx.doi.org/10.1016/j.elstat.2012.11.028

[59] D’Addio, L., Carotenuto, C., Balachandran, W., Lancia, A. \& Di Natale, F., Experimental analysis on the capture of submicron particles (PM0.5) by wet electrostatic scrubbing. Chem. Eng. Sci., 106, pp. 222-230, 2014. doi: http://dx.doi.org/10.1016/j. ces.2013.11.044

[60] Mostofi, R., Wang, B., Haghighat, F., Bahloul, A. \& Jaime, L., Performance of mechanical filters and respirators for capturing nanoparticles - limitations and future directions. Industrial Health, 48, pp. 296-304, 2010. doi: http://dx.doi.org/10.2486/indhealth.48.296

[61] Mostofi, R., Noel, A., Haghighat, F., Bahloul, A., Jaime, L. \& Cloutier, Y., Impact of two particle measurement techniques on the determination of N95 class respirator filtration performance against ultrafine particles. J. Hazard Mater., 217-218, pp. 51-57, 2012. doi: http://dx.doi.org/10.1016/j.jhazmat.2012.02.058

[62] Lin, G.-H. \& Tsai, C.-J., Numerical modeling of nanoparticle collection efficiency of single-stage wire-in-plate electrostatic precipitators. Aerosol Sci. Technol., 44, pp. 1122-1130, 2010. doi: http://dx.doi.org/10.1080/02786826.2010.512320

[63] Lin, G.-Y., Chen, T.-M. \& Tsai, C.-J., A modified Deutsch-Anderson equation for predicting the nanoparticle collection efficiency of electrostatic precipitators. Aerosol Air Qual. Res., 12, pp. 697-706, 2012. doi: http://dx.doi.org/10.4209/aaqr.2012.04.0085

[64] Long, Z. \& Yao, Q., Evaluation of various particle-charging models for simulating particle dynamics in electrostatic precipitators. J. Aerosol Sci., 41, pp. 702-718, 2010. doi: http://dx.doi.org/10.1016/j.jaerosci.2010.04.005

[65] Kim, H.-J., Han, B., Kim, Y.-J. \& Yoa, S.-J., Characteristics of an electrostatic precipitator for submicron particles using a non-metallic electrodes and collection plates. J. Aerosol Sci. 41, pp. 987-997, 2010. doi: http://dx.doi.org/10.1016/j.jaerosci.2010.08.001

[66] Poppendieck, D.G., Rim, D. \& Persily, A.K., Ultrafine particle removal and ozone generation by in-duct electrostatic precipitators. Environ. Sci. Technol., 48, pp. 2067-2074, 2014. doi: http://dx.doi.org/10.1021/es404884p

[67] Hansen, S.F., Regulation and risk assessment of nanomaterials. Too little, too late? $\mathrm{PhD}$ Thesis, Department of Environmental Engineering, Technical University of Denmark, 2009, 130 pp.

[68] Givehchi, R. \& Tan, Z., An overview of airborne nanoparticle filtration and thermal rebound theory. Aerosol Air Qual., 14, pp. 45-63, 2014. doi: http://dx.doi.org/10.4209/ aaqr.2013.07.0239

[69] Wang, J., Thompson, D. \& Hui, D.Y.H., Integrative filtration research and sustainable nanotechnology. Particuology, 11, pp. 5-13, 2013. doi: http://dx.doi.org/10.1016/j.partic.2012.06.004 
[70] Golanski, L., Guiot, A. \& Tardif, F., Experimental evaluation of individual protection devices against different types of nanoaerosols: graphite, $\mathrm{TiO}_{2}$ and Pt. J. Nanopart. Res., 12, pp. 83-89, 2010. doi: http://dx.doi.org/10.1007/s11051-009-9804-X

[71] Rensagamy, S., BerryAnn, R. \& Szalajda, J., Nanoparticle filtration performance of filtering facepieces respirators and canister/cartridges filters. J. Occup. Environ. Hyg., 10, pp. 519-525, 2013. doi: http://dx.doi.org/10.1080/15459624.2013.818229

[72] Wang, J. \& Tronville, P., Toward standardized test methods to determine the effectiveness of filtration media against airborne nanoparticles. J. Nanopart. Res., 16, pp. 1-33, 2014. doi: http://dx.doi.org/10.1007/s11051-014-2417-z

[73] Losert, S., vo Goetz, N., Bekker, C., Fransman, W., Wijnhoven, S.W.P., Delmaar, C., Hungerbuhler, K. \& Ulrich, A., Human exposure to conventional and nanoparticle-containing sprays - a critical review. Environ. Sci. Technol., 48, pp. 5366-5378, 2014. doi: http://dx.doi.org/10.1021/es5001819

[74] Debia, M., Beaudry, C., Weitchenthal, S., Tardiff, R. \& Dufresne, A., Caractérisation et contrôle de l'exposition professionnelle aux NP et particules ultra-fines, Report R-746 IRSST (Institut de Recherche en Santé et Sécurité du Travail Robert Sauvé), 2012, 66 pp.

[75] Fatisson, J., Hallé, S., Nadeau, S., Viau, C., Camus, M. \& Cloutier, Y., A pilot study towards ranking occupational health risk factors emanating from engineered nanoparticles: review of a decade of literature, Int. J. Safety Security Eng., 3, pp. 241-264, 2013. doi: http://dx.doi.org/10.2495/SAFE-V3-N4-241-263

[76] Kleindorfer, P.R. \& Kunreuther, H., Decision Sciences. An Integrative Perspective, Cambridge University Press: Cambridge, 1993, 470 pp. doi: http://dx.doi.org/10.1017/ CBO9781139173537

[77] Demou, E., Stark, W.J. \& Hellweg, S., Particle emission and exposure during nanoparticle synthesis in research laboratories. Ann. Occup. Hyg., 53, pp. 829-838, 2009. doi: http://dx.doi.org/10.1093/annhyg/mep061

[78] Tsai, S-J., Huang, R.F. \& Ellenbecker, M.J., Airborne nanoparticle exposure while using constant-flow, constant-velocity and air-curtain-isolated fume hoods. Ann. Occup. Hyg., 54, pp. 78-87, 2010. doi: http://dx.doi.org/10.1093/annhyg/mep074

[79] Lo, L.-M., Dunn, K.H., Hammond, D., Almaguer, D., Bartolomew, I., Topmiller, J., Tsai, C.-J., Ellenbecker, M. \& Huang, C.-C., Evaluation of engineering controls for manufacturing nanofiber sheets and yarns. Department of Health and Human Services Centers for Disease Control and Prevention National Institute for Occupational Safety and Health, Division of Applied Research and Technology Engineering and Physical Hazards Branch EPHB Report No. 356-11a, 2012.

[80] European Commission (b), The Appropriateness of the Risk Assessment Methodology in Accordance with the Technical Guidance Documents for New and Existing Substances for Assessing the Risks of Nanomaterials, Scientific Committee on Emerging and Newly Identified Health Risks, Brussels, Belgium, 2007, 43 pp.

[81] Asback, C., Kaminski, H., Von Barany, D., Kuhlbusch, T.A.J., Monz, C., Dziurowitz, N., Pelzer, J., Vossen, K., Berlin, K., Dietrich, S., Götz, U., Kiesling, H.-J., Schierl, R. \& Dahmann, D., Comparability of portable nanoparticle exposure monitors. Ann. Occup. Hyg., 56, pp. 606-621, 2012.

[82] Bau, S., Zimmermann, B., Rayet, R. \& Witschger, O., A laboratory study of the performance of the handheld diffusion size classifier (DiSCmini) for various aerosols in the 15-400 nm range. Environ. Sci. Processes Impacts, 17, pp. 261-269, 2015. doi: http:// dx.doi.org/10.1039/C4EM00491D 